Journal of Applied Mathematics and Bioinformatics, Vol. 10, No. 2, 2020, 39-52 ISSN: 1792-6602(print), 1792-6939(online)

https://doi.org/10.47260/jamb/1023

Scientific Press International Limited

\title{
The Convergence of Mann Iteration for Generalized $\Phi-$ hemi-contractive Maps
}

\author{
Linxin $\mathrm{Li}^{1}$, Dingping $\mathrm{Wu}^{2}$
}

\begin{abstract}
Charles[1] proved the convergence of Picard-type iterative for generalized $\Phi-$ accretive non-self maps in a real uniformly smooth Banach space. Based on the theorems of the zeros of strongly $\Phi-$ accretive and fixed points of strongly $\Phi-$ hemi-contractive we extend the results to Mann-type iterative and Mann iteration process with errors.
\end{abstract}

Keywords: strongly $\Phi-$ accretive, strongly $\Phi-$ hemi-contractive, Mann iteration process with errors, fixed point

${ }^{1}$ College of Applied Mathematics, Chengdu University of Information Technology.

${ }^{2}$ College of Applied Mathematics, Chengdu University of Information Technology.

Article Info: Received: August 21, 2020. Revised: September 8, 2020.

Published online: September 30, 2020. 


\section{Introduction}

In[2], we can see a Mann-type iteration method for a family of hemi-contractive mappings in Hilbert spaces; In[3], we can see a Halpern-Mann type Iteration for fixed point problems of a relatively nonexpansive mapping and a system of equilibrium problems; In[4], we can see that convergence of Mann's type iteration method for generalized asymptotically nonexpansive mappings; $\operatorname{In}[5]$, we can see that some Mann-type implicit iteration methods for triple hierarchical variational inequalities, systems variational inequalities and fixed point problems; In[6], we can see a Mann-type iteration method for solving the split common fixed point problem; In[7], we can see that Mann and Ishikawa-type iterative schemes for approximating fixed points of multi-valued non-self mappings.

In 2009, Charles[1] proved the convergence of Picard-type iterative for generalized $\Phi-$ accretive non-self maps in a real uniformly smooth Banach space. In this paper, we will consider to extend the result of Charles[1] to Mann-type iterative and Mann iteration process with errors.

In 1995, Liu[8] introduced what he called the Mann iteration process with errors.

In 1998, Xu[9] introduced the following alternative definitions:

Let $K$ be a nonempty convex subset of $E$ and $T: K \rightarrow K$ be any map. For any given $x_{0}, u_{0} \in K$, the process defined by

$$
x_{n+1}=a_{n} x_{n}+b_{n} T x_{n}+c_{n} u_{n}, \quad n \geq 0
$$

where $\left\{u_{n}\right\}$ is bounded sequences in $K$ and the real sequences $\left\{a_{n}\right\},\left\{b_{n}\right\},\left\{c_{n}\right\} \subset[0,1]$ satisfy the conditions

$$
a_{n}+b_{n}+c_{n}=1, \quad \forall n \geq 0 .
$$

It called the Mann iteration process with errors.

However, the most general Mann-type iterative scheme now studied is the following: $x_{0} \in K$,

$$
x_{n+1}=\left(1-c_{n}\right) x_{n}+c_{n} T x_{n}, n=0,1,2, \ldots
$$

where $\left\{c_{n}\right\}_{n=1}^{\infty} \subset(0,1)$ is a real sequence satisfying appropriate conditions (see, e.g., Chidume[10], Edelstein and O'Brian[11], Ishikawa[12]). Under the following additional assumptions (i) $\lim c_{n}=0$; and (ii) $\sum_{n=0}^{\infty} c_{n}=\infty$, the sequence $\left\{x_{n}\right\}$ 
generated by (1.2) is generally referred to as the Mann sequence in the light of Mann[13].

\section{Preliminary Notes}

Definition 2.1[1] Let $(E, \rho)$ be a metric space. A mapping $T: E \rightarrow E$ is called a contraction if there exists $k \in[0,1)$ such that $\rho(T x, T y) \leq k \rho(x, y)$ for all $x, y \in E$. If $k=1$, then $T$ is called nonexpansive.

Definition 2.2[1] Given a gauge function $\varphi$, the mapping $J_{\varphi}: E \rightarrow 2^{E^{*}}$ defined by

$$
J_{\varphi} x:=\left\{u^{*} \in E^{*}:\left\langle x, u^{*}\right\rangle=\|x\|\left\|u^{*}\right\| ;\left\|u^{*}\right\|=\varphi(\|x\|)\right\}
$$

is called the duality map with gauge function $\varphi$ where $E$ is any normed space.

In the particular case $\varphi(t)=t$, the duality map $J=J_{\varphi}$ is called the normalized duality map.

Proposition 2.3[14] If a Banach space $E$ has a uniformly Gateaux differentiable norm, then $J: E \rightarrow E^{*}$ is uniformly continuous on bounded subsets of $E$ from the strong topology of $E$ to the weak ${ }^{*}$ topology of $E^{*}$.

Definition 2.4[15] A mapping $T: E \rightarrow E$ is called strongly pseudo-contractive if for all $x, y \in E$, the following inequality holds:

$$
\|x-y\| \leq\|(1+r)(x-y)-r t(T x-T y)\|
$$

for all $r>0$ and some $t>1$. If $t=1$ in inequality(2.2), then $T$ is called pseudocontractive. As we know that $T$ is strongly pseudo-contractive if and only if

$$
\langle(I-T) x-(I-T) y, j(x-y)\rangle \geq k\|x-y\|^{2}
$$

holds for all $x, y \in E$ and for some $j(x-y) \in J(x-y)$, where $k=\frac{1}{t}(t-1) \in(0,1)$.

Definition 2.5[1] Recall that an operator $T: D(T) \subseteq E \rightarrow E$ is strongly accretive if there exists some $k>0$ such that for each $x, y \in D(T)$, there exists $j(x-y) \in J(x-y)$ such that 


$$
\langle T x-T y, j(x-y)\rangle \geq k\|x-y\|^{2} .
$$

Proposition 2.6[1] A mapping $T: E \rightarrow E$ is strongly pseudo-contractive if and only if $(I-T)$ is strongly accretive, and is strongly $\varphi$-pseudo-contractive if and only if $(I-T)$ is strongly $\varphi$-accretive. Then $T$ is generalized $\Phi$-pseudo-contractive if and only if $(I-T)$ is generalized $\Phi$-accretive.

Definition 2.7[1] Let $E$ be an arbitrary real normed linear space. A mapping $T: D(T) \subseteq E \rightarrow E$ is called strongly hemi-contractive if $F(T) \neq \varnothing$, and there exists $t>1$ such that for all $r>0$,

$$
\left\|x-x^{*}\right\| \leq\left\|(1+r)\left(x-x^{*}\right)-r t\left(T x-x^{*}\right)\right\|
$$

holds for all $x \in D(T), x^{*} \in F(T)$. If $t=1$, then $T$ is called hemi-contractive. Finally, $T$ is called generalized $\Phi-$ hemi-contractive, if for all $x \in D(T), x^{*} \in F(T)$, there exists $j\left(x-x^{*}\right) \in J\left(x-x^{*}\right)$ such that

$$
\left\langle(I-T) x-(I-T) x^{*}, j\left(x-x^{*}\right)\right\rangle \geq \Phi\left(\left\|x-x^{*}\right\|\right) .
$$

It follows from inequality (2.6) that $T$ is generalized $\Phi$-hemi-contractive if and only if

$$
\left\langle T x-x^{*}, j\left(x-x^{*}\right)\right\rangle \leq\left\|x-x^{*}\right\|^{2}-\Phi\left(\left\|x-x^{*}\right\|\right), \forall n \geq 0 .
$$

Definition 2.8[1] Let $N(T)=\{x \in E: T x=0\} \neq \varnothing$. The mapping $T: D(T) \subseteq E \rightarrow E$ is called generalized $\Phi-$ quasi-accretive if, for all $x \in E, x^{*} \in N(T)$, there exists $j\left(x-x^{*}\right) \in J\left(x-x^{*}\right)$ such that

$$
\left\langle T x-T x^{*}, j\left(x-x^{*}\right)\right\rangle \geq \Phi\left(\left\|x-x^{*}\right\|\right) .
$$

Proposition 2.9[1] If $F(T)=\{x \in E: T x=x\} \neq \varnothing$, the mapping $T: E \rightarrow E$ is strongly hemi-contractive if and only if $(I-T)$ is strongly quasi-accretive; it is strongly $\varphi$-hemi-contractive if and only if $(I-T)$ is strongly $\varphi$-quasiaccretive; and $T$ is generalized $\Phi$-hemi-contractive if and only if $(I-T)$ is generalized $\Phi$-quasi-accretive.

Proposition 2.10[1] Let $E$ be a uniformly smooth real Banach space, and let 
$J: E \rightarrow 2^{E^{*}}$ be a normalized duality mapping. Then

$$
\|x+y\|^{2} \leq\|x\|^{2}+2\langle y, J(x+y)\rangle
$$

for all $x, y \in E$.

Proposition 2.11[1] Let $\left\{\lambda_{n}\right\}$ and $\left\{\gamma_{n}\right\}$ be sequences of nonnegative numbers and $\left\{\alpha_{n}\right\}$ be a sequence of positive numbers satisfying the conditions $\sum_{n=1}^{\infty} \alpha_{n}=\infty$ and $\frac{\gamma_{n}}{\alpha_{n}} \rightarrow 0$, as $n \rightarrow \infty$. Let the recursive inequality

$$
\lambda_{n+1} \leq \lambda_{n}-\alpha_{n} \psi\left(\lambda_{n}\right)+\gamma_{n}, n=1,2, \ldots
$$

be given where $\psi:[0, \infty) \rightarrow[0, \infty)$ is strictly increasing continuous function such that it is positive on $(0, \infty)$ and $\psi(0)=0$. Then $\lambda_{n} \rightarrow 0$, as $n \rightarrow \infty$.

\section{Main Results}

In this section, we will consider to extend the result of Charles[1] to Mann-type iterative and Mann iteration process with errors under the following assumptions.

First, we extend the result of Charles[1] to Mann-type iterative.

Theorem 3.1 Suppose $K$ is a closed convex subset of a real uniformly smooth Banach space $E$. Suppose $T: K \rightarrow K$ is a bounded generalized $\Phi-$ hemicontractive map with strictly increasing continuous function $\Phi:[0, \infty) \rightarrow[0, \infty)$ such that $\Phi(0)=0$ and $x^{*} \in F(T) \neq \varnothing$. For arbitrary $x_{1} \in K$, define the sequence $\left\{x_{n}\right\}$ iteratively by

$$
x_{n+1}=\left(1-c_{n}\right) x_{n}+c_{n} T x_{n}, n=0,1,2, \ldots
$$

where $\left\{c_{n}\right\} \subseteq(0,1), \lim c_{n}=0$ and $\sum c_{n}=\infty$. Then, there exists a constant $d_{0}>0$ such that if $0<c_{n} \leq d_{0},\left\{x_{n}\right\}$ converges strongly to the unique fixed point $x^{*}$ of $T$.

Proof. Let $r$ be sufficiently large such that $x_{1} \in B_{r}\left(x^{*}\right)$. Define $G:=\overline{B_{r}\left(x^{*}\right)} \cap K$. Then, since $T$ is bounded we have that $(I-T)(G)$ is bounded. 
Let $M=\sup \left\{\left\|(I-T) x_{n}\right\|: x_{n} \in G\right\}$. As $j \quad$ is uniformly continuous on bounded subsets of $E$, for $\varepsilon:=\frac{\Phi\left(\frac{r}{2}\right)}{2 M}$, there exists a $\delta>0$ such that $x, y \in D(T),\|x-y\|<\delta$ implies $\|j(x)-j(y)\|<\varepsilon$. Set $d_{0}=\min \left\{1, \frac{r}{2 M}, \frac{\delta}{2 M}\right\}$.

Claim1: $\left\{x_{n}\right\}$ is bounded.

Suffices to show that $x_{n}$ is in $G$ for all $n \geq 1$. The proof is by induction. By our assumption, $x_{1} \in G$. Suppose $x_{n} \in G$. We prove that $x_{n+1} \in G$. Assume for contradiction that $x_{n+1} \notin G$. Then, since $x_{n+1} \in K \forall n \geq 1$, we have that $\left\|x_{n+1}-x^{*}\right\|>r$.

Thus we have the following estimates:

$$
\begin{aligned}
\left\|x_{n+1}-x^{*}\right\| & \leq\left\|x_{n}-c_{n}(I-T) x_{n}-x^{*}\right\| \\
& \leq\left\|x_{n}-x^{*}\right\|+c_{n}\left\|(I-T) x_{n}\right\| \\
& \leq r+d_{0} \cdot M \\
& \leq 2 r, \\
\left\|x_{n}-x^{*}\right\| & \geq\left\|x_{n+1}-x^{*}\right\|-c_{n}\left\|(I-T) x_{n}\right\| \\
& >r-c_{n} \cdot M \\
& >\frac{r}{2}, \quad \\
\|\left(x_{n+1}-x^{*}\right) & -\left(x_{n}-x^{*}\right)\left\|\leq c_{n}\right\|(I-T) x_{n} \| \\
& \leq c_{n} \cdot M \\
& \leq \frac{\delta}{2}<\delta,
\end{aligned}
$$

therefore,

$$
\left\|j\left(x_{n+1}-x^{*}\right)-j\left(x_{n}-x^{*}\right)\right\|<\varepsilon .
$$


Then from (3.1), the above estimates and Proposition 2.10 we have that

$$
\begin{aligned}
\left\|x_{n+1}-x^{*}\right\|^{2} & =\left\|x_{n}-c_{n}(I-T) x_{n}-x^{*}\right\|^{2} \\
& \leq\left\|x_{n}-x^{*}\right\|^{2}-2 c_{n}\left\langle(I-T) x_{n}, j\left(x_{n+1}-x^{*}\right)-j\left(x_{n}-x^{*}\right)\right\rangle \\
& -2 c_{n}\left\langle(I-T) x_{n}, j\left(x_{n}-x^{*}\right)\right\rangle \\
& \leq\left\|x_{n}-x^{*}\right\|^{2}+2 c_{n}\left\|(I-T) x_{n}\right\|\left\|j\left(x_{n+1}-x^{*}\right)-j\left(x_{n}-x^{*}\right)\right\| \\
& -2 c_{n} \Phi\left(\left\|x_{n}-x^{*}\right\|\right) \\
& \leq\left\|x_{n}-x^{*}\right\|^{2}-2 c_{n} \Phi\left(\frac{r}{2}\right)+2 c_{n} \cdot M \cdot \varepsilon \\
& \leq r^{2}+2 d_{0}\left[\frac{\Phi\left(\frac{r}{2}\right)}{2}-\Phi\left(\frac{r}{2}\right)\right] \\
& \leq r^{2}[
\end{aligned}
$$

i.e., $\left\|x_{n+1}-x^{*}\right\| \leq r$, a contradiction. Therefore $x_{n+1} \in G$. Thus by induction $\left\{x_{n}\right\}$ is bounded. Then, $\left\{T x_{n}\right\},\left\{(I-T) x_{n}\right\}$ are also bounded.

Claim2: $x_{n} \rightarrow x^{*}$.

Let $A_{n}=\left\|j\left(x_{n+1}-x^{*}\right)-j\left(x_{n}-x^{*}\right)\right\|$, Note that $x_{n+1}-x_{n} \rightarrow 0$ as $n \rightarrow \infty$ and hence by the uniform continuity of $j$ on bounded subsets of $E$ we have that

$$
A_{n} \rightarrow 0 \text { as } n \rightarrow \infty
$$

We obtain that

$$
\begin{aligned}
\left\|x_{n+1}-x^{*}\right\|^{2} & \leq\left\|x_{n}-x^{*}\right\|^{2}+2 c_{n}\left\|(I-T) x_{n}\right\|\left\|j\left(x_{n+1}-x^{*}\right)-j\left(x_{n}-x^{*}\right)\right\| \\
& -2 c_{n} \Phi\left(\left\|x_{n}-x^{*}\right\|\right) \\
& \leq\left\|x_{n}-x^{*}\right\|^{2}+2 c_{n}\left[Z_{n}-\Phi\left(\left\|x_{n}-x^{*}\right\|\right)\right] \\
& \leq\left\|x_{n}-x^{*}\right\|^{2}-2 c_{n} \Phi\left(\left\|x_{n}-x^{*}\right\|\right)+2 c_{n} Z_{n}
\end{aligned}
$$


where $Z_{n}=M A_{n} \rightarrow 0$ as $n \rightarrow \infty$.

Let $\lambda_{n}:=\left\|x_{n}-x^{*}\right\|$ and $\gamma_{n}=2 c_{n} Z_{n}$, then from inequality (3.3) we obtain that $\lambda_{n+1} \leq \lambda_{n}-2 c_{n} \Phi\left(\lambda_{n}\right)+\gamma_{n}$, where $\frac{\gamma_{n}}{c_{n}} \rightarrow 0$ as $n \rightarrow \infty$. Therefore, the conclusion of the theorem follows from Proposition 2.11.

We've done the proof of the theorem 3.1.

The following corollary follow trivially, since definition 2.5.

Corollary 3.2 Suppose $E$ is a real uniformly smooth Banach space, and $T: E \rightarrow E$ is a bounded generalized $\Phi$-accretive map with strictly increasing continuous function $\Phi:[0, \infty) \rightarrow[0, \infty)$ such that $\Phi(0)=0$ and the solution $x^{*}$ of the equation $T x=0$ exists. For arbitrary $x_{1} \in E$, define the sequence $\left\{x_{n}\right\}$ iteratively by

$$
x_{n+1}=\left(1-c_{n}\right) x_{n}-c_{n} T x_{n}, n=0,1,2, \ldots
$$

where $\left\{c_{n}\right\} \subseteq(0,1), \quad \lim c_{n}=0$ and $\sum c_{n}=\infty$. Then, there exists a constant $d_{0}>0$ such that if $0<c_{n} \leq d_{0},\left\{x_{n}\right\}$ converges strongly to the unique solution of $T x=0$.

Now, we consider to generalize to a more general case, we extend the result of Charles[1] to Mann iteration process with errors as follows.

Theorem 3.3 Suppose $K$ is a closed convex subset of a real uniformly smooth Banach space $E$. Suppose $T: K \rightarrow K$ is a bounded generalized $\Phi$-hemicontractive map with strictly increasing continuous function $\Phi:[0, \infty) \rightarrow[0, \infty)$ such that $\Phi(0)=0$ and $x^{*} \in F(T) \neq \varnothing$. For arbitrary $x_{1} \in K$, define the sequence $\left\{x_{n}\right\}$ iteratively by

$$
x_{n+1}=a_{n} x_{n}+b_{n} T x_{n}+c_{n} u_{n}, n \geq 0,
$$


where $\left\{u_{n}\right\}$ is bounded sequence in $K$ and $\left\{a_{n}\right\},\left\{b_{n}\right\},\left\{c_{n}\right\}$ are sequences in $[0,1]$ satisfying the following conditions:

(i) $a_{n}+b_{n}+c_{n}=1 \quad \forall n \geq 0 ;(i i) \sum_{n=0}^{\infty} b_{n}=\infty$;

(iii) $\lim b_{n}=0 ; c_{n}=o\left(b_{n}\right) ;(i v) \sum_{n=0}^{\infty} c_{n}<\infty$.

Then, there exists a constant $d_{0}>0$ such that if $0<b_{n}, c_{n} \leq d_{0},\left\{x_{n}\right\}$ converges strongly to the unique fixed point $x^{*}$ of $T$.

Proof. Let $r$ be sufficiently large such that $x_{1} \in B_{r}\left(x^{*}\right)$. Define $G:=\overline{B_{r}\left(x^{*}\right)} \cap K$. Then, since $T$ is bounded we have that $(I-T)(G)$ is bounded.

Let $\quad M=\max \left\{\sup \left\|(I-T) x_{n}\right\|, \sup \left\|x_{n}-u_{n}\right\|: x_{n} \in G\right\}$. As $\quad j \quad$ is uniformly continuous on bounded subsets of $E$, for $\varepsilon:=\frac{\Phi\left(\frac{r}{2}\right)}{4 M}$, there exists $a \quad \delta>0$ such that $x, y \in D(T),\|x-y\|<\delta$ implies $\|j(x)-j(y)\|<\varepsilon$.

Set $d_{0}=\min \left\{1, \frac{r}{4 M}, \frac{\delta}{4 M}, \frac{\Phi\left(\frac{r}{2}\right)}{8 M r}\right\}$.

Claim1: $\left\{x_{n}\right\}$ is bounded.

Suffices to show that $x_{n}$ is in $G$ for all $n \geq 1$. The proof is by induction. By our assumption, $x_{1} \in G$. Suppose $x_{n} \in G$. We prove that $x_{n+1} \in G$. Assume for contradiction that $x_{n+1} \notin G$. Then, since $x_{n+1} \in K \forall n \geq 1$, we have that $\left\|x_{n+1}-x^{*}\right\|>r$. Equation (3.4) becomes

$$
x_{n+1}=x_{n}-b_{n}(I-T) x_{n}-c_{n}\left(x_{n}-u_{n}\right) .
$$

Thus we have the following estimates: 


$$
\begin{aligned}
\left\|x_{n+1}-x^{*}\right\| & \leq\left\|x_{n}-x^{*}\right\|+b_{n}\left\|(I-T) x_{n}\right\|+c_{n}\left\|x_{n}-u_{n}\right\| \\
& \leq r+d_{0}(M+M) \\
& \leq 2 r, \\
\left\|x_{n}-x^{*}\right\| & \geq\left\|x_{n+1}-x^{*}\right\|-b_{n}\left\|(I-T) x_{n}\right\|-c_{n}\left\|x_{n}-u_{n}\right\| \\
> & r-d_{0}(M+M) \\
> & \frac{r}{2}, \\
\|\left(x_{n+1}-x^{*}\right) & -\left(x_{n}-x^{*}\right) \| \\
& \leq b_{n}\left\|(I-T) x_{n}\right\|+c_{n}\left\|x_{n}-u_{n}\right\| \\
& \leq 2 d_{0} M \\
& \leq \frac{\delta}{2}<\delta,
\end{aligned}
$$

therefore,

$$
\left\|j\left(x_{n+1}-x^{*}\right)-j\left(x_{n}-x^{*}\right)\right\|<\varepsilon .
$$

Then from (3.5), the above estimates and Proposition 2.10 we have that

$$
\begin{aligned}
\left\|x_{n+1}-x^{*}\right\|^{2} & =\left\|x_{n}-b_{n}(I-T) x_{n}-c_{n}\left(x_{n}-u_{n}\right)-x^{*}\right\|^{2} \\
& \leq\left\|x_{n}-x^{*}\right\|^{2}-2 b_{n}\left\langle(I-T) x_{n}, j\left(x_{n+1}-x^{*}\right)\right\rangle-2 c_{n}\left\langle x_{n}-u_{n}, j\left(x_{n+1}-x^{*}\right)\right\rangle \\
& \leq\left\|x_{n}-x^{*}\right\|^{2}+2 b_{n}\left\langle(I-T) x_{n}, j\left(x_{n+1}-x^{*}\right)-j\left(x_{n}-x^{*}\right)\right\rangle \\
& -2 b_{n}\left\langle(I-T) x_{n}, j\left(x_{n}-x^{*}\right)\right\rangle+2 c_{n}\left\langle x_{n}-u_{n}, j\left(x_{n+1}-x^{*}\right)\right\rangle \\
& \leq\left\|x_{n}-x^{*}\right\|^{2}+2 b_{n}\left\|(I-T) x_{n}\right\|\left\|j\left(x_{n+1}-x^{*}\right)-j\left(x_{n}-x^{*}\right)\right\| \\
& -2 b_{n} \Phi\left(\left\|x_{n}-x^{*}\right\|\right)+2 c_{n}\left\|x_{n}-u_{n}\right\|\left\|x_{n+1}-x^{*}\right\| \\
& \leq r^{2}+2 d_{0}\left[M \cdot \varepsilon-\Phi\left(\frac{r}{2}\right)+2 M r\right] \\
& \leq r^{2}+2 d_{0}\left[\frac{\Phi\left(\frac{r}{2}\right)}{2}-\Phi\left(\frac{r}{2}\right)\right] \\
& \leq r^{2}
\end{aligned}
$$


i.e., $\left\|x_{n+1}-x^{*}\right\| \leq r$, a contradiction. Therefore $x_{n+1} \in G$. Thus by induction $\left\{x_{n}\right\}$ is bounded. Then, $\left\{T x_{n}\right\},\left\{(I-T) x_{n}\right\}$ are also bounded.

Claim2: $x_{n} \rightarrow x^{*}$

Let $A_{n}=\left\|j\left(x_{n+1}-x^{*}\right)-j\left(x_{n}-x^{*}\right)\right\|$, Note that $x_{n+1}-x_{n} \rightarrow 0$ as $n \rightarrow \infty$ and hence by the uniform continuity of $j$ on bounded subsets of $E$ we have that

$$
A_{n} \rightarrow 0 \text { as } n \rightarrow \infty
$$

We obtain that

$$
\begin{aligned}
\left\|x_{n+1}-x^{*}\right\|^{2} & \leq\left\|x_{n}-x^{*}\right\|^{2}+2 b_{n}\left\|(I-T) x_{n}\right\|\left\|j\left(x_{n+1}-x^{*}\right)-j\left(x_{n}-x^{*}\right)\right\| \\
& -2 b_{n} \Phi\left(\left\|x_{n}-x^{*}\right\|\right)+2 c_{n}\left\|x_{n}-u_{n}\right\|\left\|x_{n+1}-x^{*}\right\| \\
& \leq\left\|x_{n}-x^{*}\right\|^{2}+2 b_{n} M \cdot A_{n}-2 b_{n} \Phi\left(\left\|x_{n}-x^{*}\right\|\right)+2 c_{n} M \cdot 2 r \\
& \leq\left\|x_{n}-x^{*}\right\|^{2}+2 b_{n}\left[M \cdot A_{n}+2 \frac{c_{n}}{b_{n}} M r-\Phi\left(\left\|x_{n}-x^{*}\right\|\right)\right] \\
& \leq\left\|x_{n}-x^{*}\right\|^{2}+2 b_{n}\left[Z_{n}-\Phi\left(\left\|x_{n}-x^{*}\right\|\right)\right] \\
& \leq\left\|x_{n}-x^{*}\right\|^{2}-2 b_{n} \Phi\left(\left\|x_{n}-x^{*}\right\|\right)+2 b_{n} Z_{n}
\end{aligned}
$$

where $Z_{n}=M A_{n}+2 \frac{c_{n}}{b_{n}} M r \rightarrow 0$ as $n \rightarrow \infty$

Let $\lambda_{n}:=\left\|x_{n}-x^{*}\right\|$ and $\gamma_{n}=2 b_{n} Z_{n}$, then from inequality (3.7) we obtain that $\lambda_{n+1} \leq \lambda_{n}-2 b_{n} \Phi\left(\lambda_{n}\right)+\gamma_{n}$, where $\frac{\gamma_{n}}{b_{n}} \rightarrow 0$ as $n \rightarrow \infty$. Therefore, the conclusion of the theorem follows from Proposition 2.11.

We've done the proof of the theorem 3.3.

The following corollary follow trivially, since definition 2.5.

Corollary 3.4 Suppose $E$ is a real uniformly smooth Banach space, and $T: E \rightarrow E$ is a bounded generalized $\Phi$-accretive map with strictly increasing 
continuous function $\Phi:[0, \infty) \rightarrow[0, \infty)$ such that $\Phi(0)=0$ and the solution $x^{*}$ of the equation $T x=0$ exists. For arbitrary $x_{1} \in E$, define the sequence $\left\{x_{n}\right\}$ iteratively by

$$
x_{n+1}=a_{n} x_{n}-b_{n} T x_{n}+c_{n} u_{n}, n \geq 0,
$$

where $\left\{u_{n}\right\}$ is bounded sequence in $K$ and $\left\{a_{n}\right\},\left\{b_{n}\right\},\left\{c_{n}\right\}$ are sequences in $[0,1]$ satisfying the following conditions:

(i) $a_{n}+b_{n}+c_{n}=1 \quad \forall n \geq 0 ;(i i) \sum_{n=0}^{\infty} b_{n}=\infty$;

(iii) $\sum_{n=0}^{\infty} b_{n}^{2}<\infty ; c_{n}=o\left(b_{n}\right) ;(i v) \sum_{n=0}^{\infty} c_{n}<\infty$.

Then, there exists a constant $d_{0}>0$ such that if $0<b_{n}, c_{n} \leq d_{0},\left\{x_{n}\right\}$ converges strongly to the unique solution of $T x=0$.

This work is supported by Applied Basic Research Foundation of Sichuan Province of China(Grant No. 2018JY0169). 


\section{References}

[1] Charles,Chidume.;Geometric Properties of Banach Spaces and Nonlinear Iterations.(2009)

[2] Nawab Hussain, Ljubomir B Ciric , Yeol Je Cho and Arif Rafiq ; On Manntype iteration method for a family of hemicontractive mappings in Hilbert spaces,Hussain et al. Journal of Inequalities and Applications 2013, 2013:41.

[3] Norimichi Hirano; A Halpern-Mann Type Iteration for Fixed Point Problems of a Relatively Nonexpansive Mapping and a System of Equilibrium Problems, Volume 2011, Article ID 632857, 22 pages.

[4] H. Zegeye,N. Shahzad;Convergence of Mann' s type iteration method for generalized asymptotically nonexpansive mappings,Computers and Mathematics with Applications 62 (2011) 4007-4014.

[5] Lu-Chuan Ceng and Xiaoye Yang;Some Mann-Type Implicit Iteration Methods for Triple Hierarchical Variational Inequalities, Systems Variational Inequalities and Fixed Point Problems,Mathematics 2019, 7, 218; doi:10.3390/math7030218.

[6] YONGHONG YAO, LIMIN LENG, MIHAI POSTOLACHE, AND XIAOXUE ZHENG;Mann-type iteration method for solving the split common fixed point problem,Journal of Nonlinear and Convex Analysis Volume 18, Number 5, 2017, 1-.

[7] Abebe R. Tufa and H. Zegeye;Mann and Ishikawa-Type Iterative Schemes for Approximating Fixed Points

[8] of Multi-valued Non-Self Mappings,Springer International Publishing 2016.

[9] Liu, L.; Ishikawa and Mann iterative process with errors for nonlinear strongly accretive mappings in Banach spaces, J. Math. Anal. Appl. 194(1995), no. 1, 114-125.

[10] Xu, Y.; Ishikawa and Mann iterative processes with errors for nonlinear strongly accretive operator equations, J. Math. Anal. Appl. 224 (1998), 91101.

[11] Chidume, C. E.; On the approximation of fifixed points of nonexpansive mappings, Houston J. Math. 7, (1981), 345-355. 
[12] Edelstein, M. and O'Brian, R. C.; Nonexpansive mappings, asymptotic regularity and successive approximations, J. London Math. Soc. 17 (1978), no. $3,547-554$.

[13] Ishikawa, S.; Fixed points and iteration of nonexpansive mapping in a Banach space, Proc. Amer. Math. Soc. 73 (1976), 61-71.

[14] Mann, W. R.; Mean value methods in iteration, Proc. Amer. Math. Soc. 4 (1953), 506-510.

[15] Cudia, D. F.; The theory of Banach spaces: Smoothness, Trans. Amer. Math. Soc. 110 (1964), 284-314.

[16] Browder, FE: Nonlinear operators and nonlinear equations of evolution in Banach spaces. In: Proc. Of Symposia in Pure Math., Vol. XVIII, Part 2 (1976). 